\title{
Are natural killer cells the key to treating Epstein-Barr virus-associated lymphoproliferative disorders?
}

\author{
Michael J. Robertson
}

Bone Marrow and Stem Cell Transplantation Program, Division of Hematology and Oncology, Department of Medicine, Indiana University School of Medicine, Indianapolis, Indiana, USA

Address correspondence to: Michael J. Robertson, Bone Marrow and Stem Cell Transplantation Program, Indiana University Medical Center, 1044 West Walnut Street, Room R4-202, Indianapolis, Indiana 46202, USA.

Phone: (317) 274-6843; Fax: (317) 278-2262; E-mail: mjrobert@iupui.edu.

J. Clin. Invest. 108:801-802 (2001). DOI:10.1172/JCI200114003.

Epstein-Barr virus (EBV) is a human herpes virus that infects predominantly oropharyngeal epithelial cells and mature B lymphocytes (1). Infection of epithelial cells by EBV results in replication of linear viral DNA, production of new virions, and lysis of the infected cell. In contrast, when B cells become infected by EBV, the virus can persist in a latent form and the cells can become immortalized and survive for the lifetime of the host. IgG antibodies specific for EBV, which indicate previous EBV infection, are found in the blood of more than $90 \%$ of adults. Primary EBV infection in children usually results in asymptomatic seroconversion or mild nonspecific symptoms, whereas about $50 \%$ of adolescents and adults with acute EBV infection experience infectious mononucleosis.

Primary EBV infection resolves without apparent clinical sequelae in virtually all immunocompetent people. $\mathrm{CD}^{+}$cytotoxic $\mathrm{T}$ lymphocytes (CTLs) appear to be crucial for control of the transformed B cells during primary EBV infection, although natural killer (NK) cells and other effector cells may participate in the immune response to $\operatorname{EBV}(1,2)$. EBV-specific memory CD8 ${ }^{+}$ $\mathrm{T}$ cells are believed to be primarily responsible for long-term in vivo suppression of latently infected B cells. EBV seropositive persons who become immunocompromised are at risk for the development of EBV-associated lymphoproliferative disorders (EBVLPDs) (1, 2). EBV-LPDs have been observed in patients with HIV infection and patients on immunosuppression after allogeneic solid organ or hematopoietic stem cell transplantation. EBV-LPDs are clinically and pathologically heterogeneous $(3,4)$. A spectrum of histopathologic appearances, from polyclonal B cell hyperplasia to monoclonal diffuse aggressive $B$ cell non-Hodgkin lymphoma, can be seen in EBV-LPD.

Treatment of EBV-LPDs is currently unsatisfactory. EBV-LPD regresses in some patients after the reduction or discontinuation of therapeutic immunosuppression $(1,2)$. However, this approach exposes patients to risks of life-threatening organ failure or graftversus-host disease (GVHD). Conventional chemotherapy and/or radiotherapy are sometimes effective but can be quite toxic in this clinical setting (5). In contrast, treatment with anti-B cell mAb's appears to be very well tolerated $(6,7)$, although it may not be sufficient for patients with widely disseminated or more aggressive disease. Infusion of $T$ cells from an HLA-compatible, EBVseropositive donor can reconstitute EBV-specific immunity in immunocompromised recipients (8). Infusion of unfractionated donor leukocytes puts patients at risk for GVHD, however, and EBV-specific donor $T$ cell lines (9) are arduous to prepare and are not widely available. Thus, novel therapeutic approaches are clearly needed for patients with EBV-LPD.

Lack of suitable animal models has been a major obstacle to the development of new treatments for EBV-LPD. However, the ability to reconstitute a human immune system in SCID mice offers a promising system for the study of this disease. Mature human T, $B$, and NK cells can persist and function in this mouse strain, which lacks endogenous $\mathrm{T}$ and $\mathrm{B}$ lymphocytes due to a defect in the recombinase system required for productive $T$ cell receptor or immunoglobulin gene rearrange- ment (10). Injection of normal human peripheral blood lymphocytes (huPBLs) into SCID mice yields lymphohematopoietic chimeras, which have proved of value in studies of cancer and infectious diseases $(11,12)$. More recently, several groups have created hu-PBL-SCID chimeras using PBLs from EBV-seropositive donors and have shown that recipient mice reproducibly develop a fatal lymphoproliferative disorder of human $B$ cells that resembles human EBV-LPD (13-15). The hu-PBL-SCID mouse would therefore appear to provide an excellent system in which to test immunotherapeutic approaches to EBV-LPD.

In this issue of the JCI, Baiocchi et al. (16) describe effective cytokine-based immunotherapy for EBV-LPD in this mouse model. Systemic administration of recombinant human GM-CSF and low-dose IL-2 was found to delay the development of EBV-LPD and prolong the survival of hu-PBL-SCID mice that were depleted of murine NK cells. The survival of hu-PBL-SCID mice treated with either IL-2 alone or GM-CSF alone was markedly inferior to that of mice treated with both cytokines (16) and was similar to that of animals treated with placebo (17). Experiments using PBLs depleted of various leukocyte subsets demonstrated that human NK cells, CD8 T cells, and monocytes contributed to the protective effect of IL-2 and GM-CSF. Moreover, an expanded population of EBV-specific human $\mathrm{CD}^{+} \mathrm{T}$ cells was detected in the spleens of hu-PBLSCID mice treated with IL-2 and GMCSF in combination, but not of mice treated with IL-2 alone. These results indicate that treatment with IL-2 and GM-CSF promotes the expansion of 
EBV-specific $\mathrm{CD}^{+} \mathrm{T}$ cells and suppresses the proliferation of EBV-transformed human B cells in vivo.

Several important issues are raised by the provocative findings of Baiocchi et al. (16). EBV antigen-specific $\mathrm{CD}^{+}$ $T$ cells have been thought to be the most important effectors for the control of EBV-transformed B cells (18), but the present data demonstrate a crucial role for NK cells in cytokine-based therapy for EBV-LPD. Without exception, animals depleted of both human and murine NK cells succumbed to EBV-LPD despite combined treatment with IL-2 and GM-CSF. Thus, the presence of NK cells is absolutely required for successful treatment of EBV-LPD in this model. In contrast, $20 \%$ of mice treated with both cytokines survived despite depletion of human CD8 T cells or monocytes. Currently it is not clear whether human NK cell-depleted mice fail to develop EBV-specific $\mathrm{CD}^{+}$ $T$ cells, which would suggest a requirement for NK cells in the afferent arm of the adaptive immune response. Alternatively, the animals may succumb to EBV-LPD despite the presence of antigen-specific $\mathrm{T}$ cells if NK cells are required along with CTLs in the effector phase of the immune response. One could begin to dissect the contribution of NK cells to the control of EBV-LPD in this model by evaluating their role in the afferent and efferent phases of EBVspecific CD8 T cell immunity.

The task of elucidating the role of NK cells in the hu-PBL-SCID model of EBV-LPD is complicated by the presence of functional murine NK cells in SCID mice (19). When hu-PBL-SCID mice are not depleted of murine NK cells, treatment with low-dose IL-2 alone is effective against EBV-LPD, and treatment with GM-CSF is not required (17). The mechanism whereby GM-CSF administration compensates for the absence of endogenous murine NK cells is not known and deserves further investigation. A clue may be offered by previous work of Baiocchi and Caligiuri (17), who found that successful treatment by low-dose IL-2 alone was associated with an accumulation of murine monocytes, murine NK cells, and human NK cells in peritoneal cavity of hu-PBL-SCID mice. Depletion of murine NK cells led to fatal EBV-LPD in $100 \%$ of IL-2-treated mice, whereas depletion of human NK cells had no effect. It seems reasonable to hypothesize that murine monocytes could interact productively with murine NK cells, but not human NK cells, in a protective response to EBVtransformed $B$ cells. The accumulation of human monocytes as well as human NK cells in the peritoneal cavity of huPBL-SCID mice treated with both IL-2 and GM-CSF might compensate for the absence of murine NK cells in this system. Testing these hypotheses is conceptually straightforward and should shed further light on the mechanisms by which NK cells help to control EBV-LPD.

The attractive features of the hu-PBLSCID experimental system should not blind one to its limitations (20). The immune defects that allow EBV-transformed B cells to proliferate unchecked in hu-PBL-SCID chimeras (15) may be very different from those of most immunocompromised humans. It is obvious, for example, that the human lymphocyte subsets that are adoptively transferred to SCID mice by injection of PBLs from healthy EBV-seropositive donors are not the same as the endogenous lymphocyte subsets of patients with advanced HIV infection. These issues become particularly acute when one wishes to use the results of huPBL-SCID experiments to design therapeutic approaches for human disease. For example, if GM-CSF in the huPBL-SCID model of Baiocchi et al. (16) is required merely to enhance the survival of injected human monocytes in the murine peritoneal cavity, administration of GM-CSF may not be necessary for the treatment of EBV-LPD in humans. Nevertheless, the results of Baiocchi and colleagues $(16,17)$ provide a solid foundation for further studies that can elucidate the mechanisms by which NK cells, monocytes, and $\mathrm{CD}^{+} \mathrm{T}$ cells contribute to the control of EBV-transformed human B cells in vivo. It is to be hoped that such preclinical studies will ultimately lead to more effective treatment for immunocompromised patients afflicted with EBV-LPD.

\section{Acknowledgments}

The author is supported in part by NIH grant MO1 RR00750-2753.

1. Cohen, J.I. 2000. Epstein-Barr virus infection. N Engl. J. Med. 343:481-492.

2. Purtilo, D.T., Strobach, R.S., Okano, M., and
Davis, J.R. 1992. Epstein-Barr virus-associated lymphoproliferative disorders. Lab. Invest. 67:5-23. 3. Knowles, D.M., et al. 1995. Correlative morphologic and molecular genetic analysis demonstrates three distinct categories of posttransplantation lymphoproliferative disorders. Blood. 85:552-565.

4. Orazi, A., et al. 1997. Posttransplantation lymphoproliferative disorders in bone marrow transplant recipients are aggressive diseases with a high incidence of adverse histologic and immunobiologic features. Am. J. Clin. Pathol. 107:419-429.

5. Mamzer-Bruneel, M.-F., et al. 2000. Durable remission after aggressive chemotherapy for very late post-kidney transplant lymphoproliferation: a report of 16 cases observed in a single center. $J$. Clin. Oncol. 18:3622-3632.

6. Fischer, A., et al. 1991. Anti-B-cell monoclonal antibodies in the treatment of severe B-cell lymphoproliferative syndrome following bone marrow and organ transplantation. N. Engl. J. Med. 324:1451-1456

7. Kuehnle, I., et al. 2000. CD20 monoclonal antibody (rituximab) for therapy of Epstein-Barr virus lymphoma after hematopoietic stem-cell transplantation. Blood. 95:1502-1505.

8. Papadopoulos, E.B., et al. 1994. Infusions of donor leukocytes to treat Epstein-Barr virus-associated lymphoproliferative disorders after allogeneic bone marrow transplantation. N. Engl. J. Med. 330:1185-1191.

9. Rooney, C.M., et al. 1998. Infusion of cytotoxic T cells for the prevention and treatment of EpsteinBarr virus-induced lymphoma in allogeneic transplant recipients. Blood. 92:1549-1555.

10. Bosma, G.C., Custer, R.P., and Bosma, M.J. 1983. A severe combined immunodeficiency mutation in the mouse. Nature. 301:527-530.

11. Mosier, D.E., Gulizia, R.J., Baird, S.M., and Wilson, D.B. 1988. Transfer of functional human immune system to mice with severe combined immunodeficiency. Nature. 335:256-259.

12. McCune, J.M., et al. 1988. The SCID-hu mouse: murine model for the analysis of human hematolymphoid differentiation and function. Science. 241:1632-1639.

13. Cannon, M.J., Pisa, P., Fox, R.I., and Cooper, N.R 1990. Epstein-Barr virus induces aggressive lymphoproliferative disorders of human B cell origin in SCID/hu chimeric mice. J. Clin. Invest. 85:1333-1337.

14. Rowe, M., et al. 1991. Epstein-Barr Virus (EBV)associated lymphoproliferative disease in the SCID mouse model: implications for the pathogenesis of EBV-positive lymphomas in man. $J$. Exp. Med. 173:147-158.

15. Veronese, M.L., et al. 1992. Lymphoproliferative disease in human peripheral blood mononuclear cell-injected SCID mice. I. T lymphocyte requirement for B cell tumor generation. J. Exp. Med. 176:1763-1767.

16. Baiocchi, R.A., et al. 2001. GM-CSF and IL-2 induce specific cellular immunity and provide protection against Epstein-Barr virus lymphoproliferative disorder. J. Clin. lnvest. 108:887-894.

17. Baiocchi, R.A., and Caligiuri, M.A. 1994. Lowdose interleukin 2 prevents the development of Epstein-Barr virus (EBV)-associated lymphoproliferative disease in scid/scid mice reconstituted i.p. with EBV-seropositive peripheral blood lymphocytes. Proc. Natl. Acad. Sci. USA. 91:5577-5581.

18. Rickinson, A.B., and Moss, D.J. 1997. Human cytotoxic T lymphocyte responses to Epstein-Barr virus infection. Annu. Rev. Immunol. 15:405-431.

19. Dorshkind, K., Pollack, S.B., Bosma, M.J., and Phillips, R.A. 1985. Natural killer (NK) cells are present in mice with severe combined immunodeficiency (scid). J. Immunol. 134:3798-3801.

20. Tary-Lehmann, M., Saxon, A., and Lehman, P.V. 1995. The human immune system in hu-PBLSCID mice. Immunol. Today. 16:529-533. 